\title{
The problem of the electrical discharge in an internally wired capacitor
}

\author{
O problema da descarga elétrica um capacitor internamente conectado
}

\author{
F. B. Rizzato* \\ Instituto de Física, Universidade Federal do Rio Grande do Sul, Porto Alegre, RS, Brasil
}

Recebido em 18 de março de 2016. Revisado em 26 de abril de 2016. Aceito em 27 de abril de 2016

\begin{abstract}
The present work investigates the dynamics of electrical discharges in a capacitor where both plates are internally connected by a conducting wire. A simplifying assumption used to solve the problem is to consider a uniform distribution of the surface charges on the plates. This implies uniform electric fields as well, and allows to calculate the current in the wire as if it were flowing along a simple RC circuit. We show that although the assumption is good if the conductivity of the wire is small, it fails if the conductivity becomes larger than a critical value. The device's behavior beyond this critical value is then analyzed and discussed. The relevance of the problem is discussed in connection to solid-state devices, as well as in problem solving contexts.
\end{abstract}

Keywords: Electrical discharges, Vacuum and solid-state electronics, Electromagnetic waves.

O presente trabalho investiga a dinâmica de descargas elétricas em um capacitor onde ambas as placas estão internamente conectadas por um fio condutor. Uma suposição simplificadora usada para resolver o problema é a de considerar uma distribuição uniforme de cargas superficiais nas placas. Isto implica em campos elétricos também uniformes, e permite calcular a corrente no fio como se estivesse fluindo ao longo de um circuito RC simples. Mostramos que embora a suposição seja boa se a condutividade do fio é pequena, falha se a condutividade ultrapassa um valor crítico. O comportamento do dispositivo além deste valor crítico é então analisado e discutido. A relevância do problema é discutida em conexão a dispositivos de estado sólido, bem como em contextos relacionados à resolução de problemas.

Palavras-chave: Descargas elétricas, Eletrônica de vácuo e de estado sólido, Ondas eletromagnéticas.

\section{Introduction}

One problem occasionally discussed in electromagnetism classes is that of the electrical discharge in an internally wired capacitor [1-3]. That is, a capacitor whose plates are connected directly one to another by a resistive wire, and not indirectly connected across an external electronic circuit as is the usual situation. The setting is not only of relevance in problem solving contexts, but also in solid-state device electronics, where leakage currents may flow from one plate to the other along faulty inner channels of solid-state planar capacitors [4.

*Endereço de correspondência: rizzato@if.ufrgs.br
The discharge dynamics is of peculiar interest because it involves simultaneous fluxes of electric and displacement field currents inside the capacitor, as opposed to the usual model of isolated plates which admits only displacement currents if the circuit is set to operate under nonstationary regimes $[5]$. The simultaneous presence of electric and displacement currents in the same volume provides one convenient setting to discuss the Ampère's law, in its form corrected by Maxwell, since the total current flowing inside the capacitor must satisfy continuity, the very condition that motivated Maxwell's analysis. Furthermore, adequate manipulation of electric and displacement currents allows the evaluation of the magnetic field inside the capacitor, which is often 
the primary question posed in theoretical discussions and which will be used in the present analysis to pose proper boundary conditions.

The usual approach to accomplish the task of calculating the fields inside the capacitor is to assume circular geometry, uniformly distributed surface charges over both plates at all times, and a resistive wire connecting both geometric centers of the plates. Under the condition of uniform electric fields, which is critical for reliability of solid-state devices, and with help of the capacitive relationship between electric field and surface charges, one can calculate: (i) the current flowing along the wire, (ii) the resulting charge depletion over the plates along with the associated variation of the electric field and, ultimately, (iii) the magnetic field as obtained from the Ampère's law 1,2].

The issue with this kind of approach is precisely the assumptions on uniformity. As pointed out by Feynman, in an analysis excluding the wire and the associated electric current, if there is dynamical flux of magnetic field, there must be space dependence of the electric field because Faraday-Lenz's law demands so [6.7]. In other words, the assumptions of a uniform electric field must be examined with care.

Feynman goes on and obtains a solution for the unwired case in terms of Bessel's functions [8]. His solution is fully self-consistent, admits boundary conditions, and is in fact what motivates the present analysis. More to the point, we wish to examine what happens with a discharge in the internally wired capacitor when we allow for space-time effects. In particular, we would like to examine how good is the usual assumption of a thorough uniform electric field inside the device during the discharge.

The paper is organized as follows: in $\S 2$ we introduce the model to be studied; in $\S 3$ an analytical and numerical investigation is carried out; and in $\S 4$ we draw our final conclusions.

\section{The model}

The model we shall employ, as represented in Fig. 1. is almost self-explanatory. Two perfectly conducting circular plates, both of radius $a$, are connected through a cylindrical solid structure of radius $b<a$ and length $L$. The cylindrical structure is a material of uniform conductivity $g$ which is defined in terms of the electric current density $\mathbf{j}$ and electric field $\mathbf{E}$ as $\mathbf{j}=g \mathbf{E}$. The conducting region is somewhat

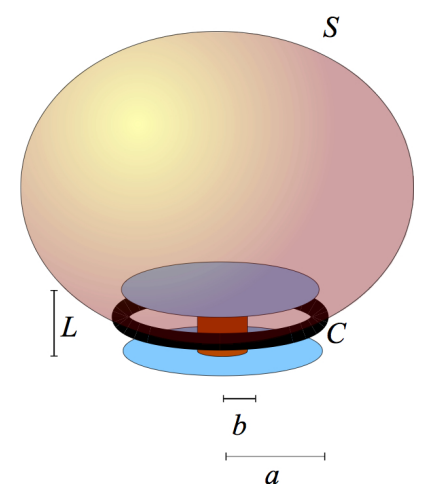

Figure 1: Relevant ingredients and geometry of the internally wired capacitor. Both plates have radius $a$ and the wire has radius $b$. The role of surface $S$ bounded by curve $C$ is discussed in the text.

equivalent to lossy dielectric regions in electronic devices [9].

The evolution law of the electromagnetic field in the volume between the plates follows from the time dependent Maxwell's equations

$$
\begin{array}{r}
\frac{\partial \mathbf{B}}{\partial t}=-\nabla \times \mathbf{E}, \\
\nabla \times \mathbf{B}=\mu_{0} \mathbf{j}+\frac{1}{c^{2}} \frac{\partial \mathbf{E}}{\partial t},
\end{array}
$$

with $\mathbf{B}$ as the magnetic field, $\mu_{0}$ as the vacuum permeability, and $c$ as the speed of light. The pair of equations (1) and (2) can be combined into a wave equation with a current term

$$
\nabla^{2} \mathbf{E}-\frac{1}{c^{2}} \frac{\partial^{2} \mathbf{E}}{\partial t^{2}}-\mu_{0} \frac{\partial \mathbf{j}}{\partial t}=0
$$

if one uses $\nabla \cdot \mathbf{B}=0$ and assumes no charge build-up between the capacitor plates so that $\nabla \cdot \mathbf{E}=0$ as well.

In the following we use the small gap condition $L \ll a$, as roughly suggested by the aspect ratio of Fig. 1. Under this circumstance we neglect fringe effects and also consider the case of uniform fields along the axis, noting that in this case the length $L$ has no functional bearing on the analysis to be performed. However, we allow for full azimuthally symmetric dependence on the radial coordinate $r$ of a cylindrical set of coordinates, which precisely embodies the kind of nonuniformity to be studied in this work.

Taking the polarization of the fields into account, the electric and magnetic fields are repectively represented by

$$
\mathbf{E}(r, t)=E(r, t) \hat{z}
$$


and

$$
\mathbf{B}(r, t)=B(r, t) \hat{\theta},
$$

with the hatted quantities as the polarization versors for the respective fields. The underlying adopted geometry is consistent with the divergence-less electric field and promptly leads to the following equation for the scalar field $E(r, t)$ in the form

$$
\begin{aligned}
& \frac{1}{r} \frac{\partial}{\partial r}\left(r \frac{\partial E(r, t)}{\partial r}\right)-\frac{\partial^{2} E(r, t)}{\partial t^{2}} \\
& -H(b-r) g \frac{\partial E(r, t)}{\partial t}=0 .
\end{aligned}
$$

In Eq. (6), the Heaviside step function $H(x \geq 0)=$ $1, H(x<0)=0$ controls the presence of the electric current between the plates; current can only flow in the region $r<b$. In addition, space, time and the conductivity have all been made respectively dimensionless with $r / a \rightarrow r, c t / a \rightarrow t$, and $\mu_{0} g c a \rightarrow$ $g$.

\section{Solutions for the wave equation}

\subsection{Analytical approach}

We look for solutions of the wave equation (6) in a normal mode separable form [10],

$$
E(r, t)=E_{0} e^{-i \omega t} \psi_{\omega}(r)
$$

The normal mode frequency $\omega$ and the spatial eigenfunction $\psi_{\omega}$ should be sought-for with help of the differential equation and its boundary conditions which we now discuss. We start off with the boundary conditions, and analyze the differential equation and the appropriate continuity conditions immediately afterwards.

Solutions must be finite at any radial position, particularly at the origin, so

$$
\psi_{\omega}(r \rightarrow 0)<\infty .
$$

Furthermore, we recall that fringe effects are absent and avoid the inherent complications of an open boundary problem assuming that most of the electric field lines reside between both plates. Under this approximation, the total current (electric plus displacement) flux crossing the open surface $S$ bounded by circular curve $C$ of radius $r=1$ in Fig. (1) vanishes. Likewise, the magnetic field vanishes at $r=1$ since its line integral along $C$ equals the total current flux across $S$ from Ampère's law. From expressions (1) and (4) the magnetic field is seen to be proportional to the simple derivative of the electric field $E(r, t)$. Therefore, from expression (7) our boundary condition at $r=1$ reads

$$
\left.\frac{\partial \psi_{\omega}}{\partial r}\right|_{r=1}=0
$$

Let us then resolve the temporal part of Eq. (6) and write down the differential equation in its spatial form both in the conducting and vacuum regions. One has

$\frac{1}{r} \frac{\partial}{\partial r}\left(r \frac{\partial \psi_{\omega}}{\partial r}\right)+\left\{\begin{aligned} & \kappa_{\text {in }}^{2} \psi_{\omega}=0 \quad \text { for } \quad r \leq b, \\ & \text { with } \quad \kappa_{\text {in }}^{2} \equiv \omega^{2}+i g \omega, \\ & \kappa_{\text {out }}^{2} \psi_{\omega}=0 \text { for } r>b, \\ & \text { with } \kappa_{\text {out }}^{2} \equiv \omega^{2},\end{aligned}\right.$

where we recall that both magnetic and electric fields are continuous at $r=b$. In other words, both $\psi_{\omega}$ and its space derivative must be continuous across the wire radius. We note that the vacuum wave vector $\kappa_{\text {out }}$ coincides with the frequency $\omega$.

Both equations contained in the above set (10) are in the form of azimuthally symmetric Bessel's differential equations, whose solutions can be formed by appropriate linear combinations of Bessel's functions of first and second kind, $J_{0}$ and $Y_{0}$ respectively [11].

In the conduction region, where $r$ can go to zero, one must pick the $J_{0}$ function, which is finite at the origin. In the vacuum the complete solution must be formed as a linear combination of the $J_{0}$ and $Y_{0}$ functions. One thus has

$$
\psi_{\omega}(r)= \begin{cases}J_{0}\left(\kappa_{\text {in }} r\right) & \text { if } \quad r \leq b, \\ \alpha J_{0}\left(\kappa_{\text {out }} r\right)+\beta Y_{0}\left(\kappa_{\text {out }} r\right) & \text { if } \quad r>b,\end{cases}
$$

where $\psi_{\omega}$ is conveniently scaled that $\psi_{\omega}(r=0)=1$, and where we see that the Bessel's functions depend on complex arguments.

The boundary condition (9) along with the derivative properties of the Bessel's functions, $J_{0}^{\prime}(x)=$ $-J_{1}(x) ; Y_{0}^{\prime}(x)=-Y_{1}(x)[8]$, yields

$$
\beta=-\alpha \frac{J_{1}\left(\kappa_{\text {out }}\right)}{Y_{1}\left(\kappa_{\text {out }}\right)},
$$

and combination of relations $(10)$ and 111 with the continuity of function $\psi_{\omega}$ and its derivative at $r=b$ finally generates relation 


$$
\frac{J_{0}\left(\kappa_{\text {in }} b\right)}{J_{1}\left(\kappa_{\text {in }} b\right)}=\frac{\kappa_{\text {in }}}{\kappa_{\text {out }}} \frac{Y_{1}\left(\kappa_{\text {out }}\right) J_{0}\left(\kappa_{\text {out }} b\right)-J_{1}\left(\kappa_{\text {out }}\right) Y_{0}\left(\kappa_{\text {out }} b\right)}{Y_{1}\left(\kappa_{\text {out }}\right) J_{1}\left(\kappa_{\text {out }} b\right)-J_{1}\left(\kappa_{\text {out }}\right) Y_{1}\left(\kappa_{\text {out }} b\right)}
$$

from which one can determine the spectrum of allowed values of $\omega$.

\subsection{Numerical analysis of the frequency spectrum}

We have two free parameters in the dimensionless system: the conductivity $g$ and the radius $b$ of the inner, conductive region. In order to emulate a thin conducting wire, we shall take $b=0.1$ unless otherwise stated. Given the fixed radius $b$, we shall then examine the imaginary and real parts of the complex frequency $\omega=\omega(g)$ as functions of $g$.

If we took a uniform medium, characterized by $g=0$, solutions would be given in the form of single Bessel functions of the first kind $J_{0}\left(\kappa_{n} r\right)$ throughout the entire volume of the capacitor, with $\kappa_{n}$ as one of the roots of expression $\left.J_{0}^{\prime}\left(\kappa_{n} r\right)\right|_{r=1}=-\kappa_{n} J_{1}\left(\kappa_{n}\right)=$ 0 . One particular solution would be the one with no spatial dependence, $\kappa_{0}=0$. The dispersion relation $\omega=\kappa$ for this $g=0$ case would imply a vanishing frequency $\omega$ as well, which places this particular setting in a fully stationary regime where the capacitor plates remain uniformly and invariantly charged.

We may start our numerical investigation at this point, since our central question is precisely on how far does the discharge remain approximately homogeneous as the conductivity increases. Given the initial root $\kappa_{0}=\omega(g=0)=0$ we will iteratively calculate the corrected frequency $\omega=\omega(g)$ as $g$ departs from zero. We shall also extend the technique to the adjacent smallest nontrivial real root $\kappa_{n=1}>0$ to have a more panoramic view of the system behaviour even when the zero conductivity case is neither homogeneous nor stationary.

Figure 2 reveals the behaviour of the imaginary and real parts of the complex frequency $\omega(g)$ as functions of $g$ (only the root with positive real part is depicted in each panel).

As mentioned, panel (a) is the one of central interest in our discussion, since it describes the frequency as it rises from the fully stationary and uniform case $\omega(g=0)=0$. One indeed sees that at relatively small values of $g$ the real part of the frequency stays glued the zero frequency axis, while its modular imaginary part rises very slowly away from the axis. (a)

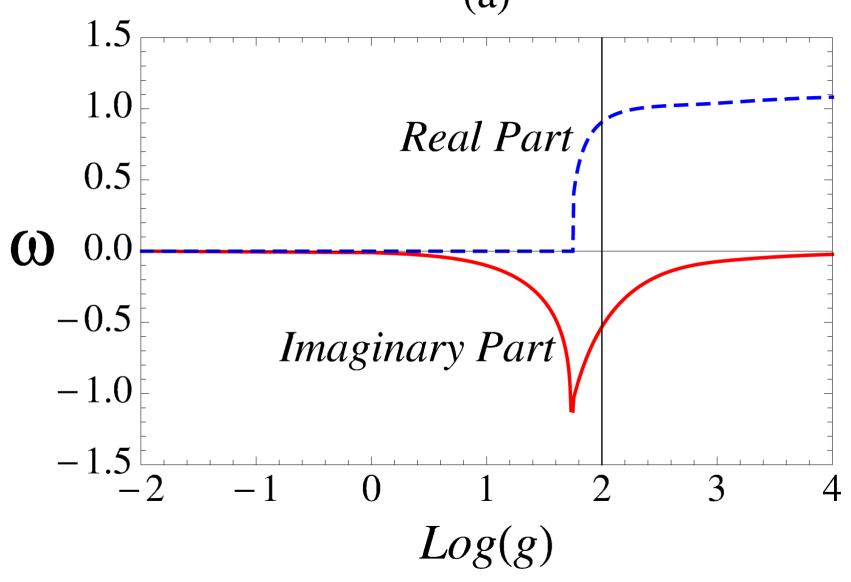

(b)

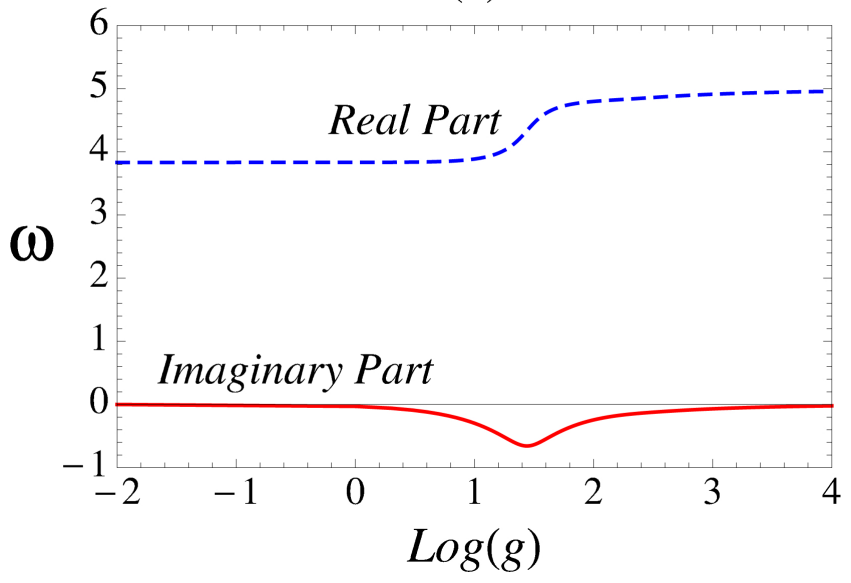

Figure 2: Real and imaginary parts of the eigen-frequencies of the discharge modes. In panel (a) with start from $\omega(g=$ $0)=0$ and in panel (b) from the lowest nontrivial root of $J_{1}(\kappa)=0$, which is approximately $\omega(g=0)=3.831$. The vertical line in (a) locates the respective $g_{\text {transition }}$ as estimated by Eq. (13).

This kind of behaviour goes on until a sharply defined point where the real part starts to increase while the modular imaginary part starts to decrease. This sharply defined point may appear unexpected at a first look, but a closer examination shows that it must exist. Indeed, when $g$ lies within its range of small values $g \ll 1$, there is little difference between the conducting region $r \leq b$ and vacuum region $r>b\left[\begin{array}{l|l}12 & 14]\end{array}\right.$. The electric field is thus mostly uni- 
form and slowly varying, and the current basically grows as the conductivity increases. The decay time scale for the electrical discharge acquires a large but finite value and the modular imaginary part of the negative imaginary frequency, which can be thought as the inverse of the discharge time scale, increases slightly. But this kind of behaviour cannot go on indefinitely. If $g$ becomes too large, skin depth effects expel the electric field from the conducting region, dissipation ceases [15], and the system re-morphs into a resonant cavity which extends from $r=b$ to $r=1$ and supports a real resonant frequency. This is why, as seen in panel (a), the real part of the frequency saturates at its terminal value and the imaginary part approaches a final vanishing value.

Estimates can be even obtained for the transition point. First of all one should notice that for small conductivities, the time scale of the electrical discharge can be evaluated as the characteristic time $\tau$ of a RC circuit composed by the capacitor and the resistive wire connecting the plates: one has $|\omega| \equiv 1 / \tau \sim 1 / R C \sim g b^{2}$ with $R$ and $C$ respectively as the resistance and capacitance of the RC circuit. Secondly, one can estimate the already mentioned skin depth characteristic length $\delta_{s}$ associated with the penetration of the electric field lines into the conducting portion of the circuit: $\delta_{s} \sim 1 / \sqrt{g|\omega|}[10$. For very small values of either $g$ or $|\omega|$, the skin depth is large and the field freely diffuses into the wire. For large values of the combination $g|\omega|$, on the other hand, $\delta_{s}$ diminishes to a point where it becomes smaller than $b$. This is where the field begins to be expelled from the wire as commented before. The critical $g$ can be estimated as one takes $\delta_{s} \sim b$ and makes use of $|\omega| \equiv 1 / \tau$ above. One obtains

$$
g_{\text {transition }} \sim 1 / b^{2},
$$

which well approximates the transition in the case $b=0.1$ seen in panel (a) of Fig. 2 - $g_{\text {transition }}$ is indicated by the vertical black line. We have further tested expression (13) for $b=0.01$ and once again nice agreement is obtained. We point out that the transition seen in Fig. 1(a) is similar to the one seen in RLC circuits, whenever a dominant $\mathrm{RC}$ mode jumps to an LC dominant mode.

To make a comparison with an inherently nonuniform case, in panel (b) we examine the behaviour evolving from the first nontrivial oscillatory mode associated with the $g=0$ case. More precisely, we pick $\kappa_{1}$ with $J_{1}\left(\kappa_{1}\right)=0$ and analyse what happens as $g$ increases. (a)

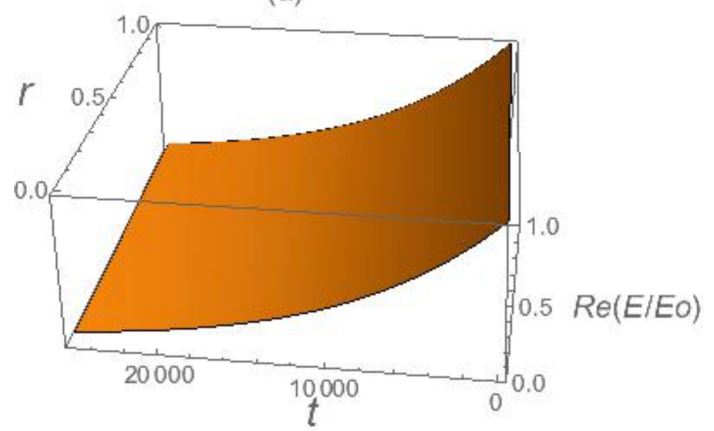

(b)

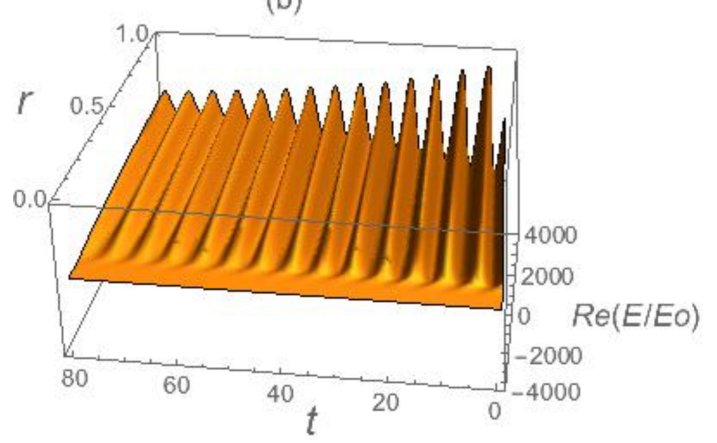

Figure 3: Space-time history for the eigenmodes of the internally wired capacitor: $g=0.01$ in panel (a) and $g=10^{4}$ in panel (b). For small conductivities panel (a) shows that the discharge follows the decay pattern of a simple RC circuit, while for large conductivities panel (b) shows that the system behaves as a weakly dissipative resonant cavity.

The result is depicted in the curves of panel (b) which essentially shows the same kind of profile than panel (a), with the exception of the expected offset associated with the nonzero frequency at $g=0$; $\omega(g=0)=3.831$.

To complement the present analysis, Fig. 3 offers two panels displaying the space-time history of the real part of the electric field in two conductive regimes: small conductivity $g=0.01$ in (a) and large conductivity $g=10^{4}$ in (b), both roots arising from the $\kappa_{0}=0$ case.

While the first panel, panel (a), shows the nearly uniform decay of the electric field towards its vanishing final value, the second panel, panel (b), reveals what is best described as a very slow decay of an eigenmode of a high quality resonant cavity affected by a truly small dissipation rate. In particular, we point out how much smaller is the normalized field $E / E_{0}$ inside the conducting wire, within $r<b$, as compared with the field without. We also point out that since the electric field is flat inside the wire, the associated magnetic field appears for the first time only at its surface. 


\section{Conclusions}

In the present work we performed a fully self-consistent electromagnetic analysis of a device used to give students a firm grasp on the role of displacement currents in Maxwell's theory. The model is built-up on the concept of an internally wired capacitor where the plates are directly connected one to another as seen in Fig. 1 .

The model is also suggested to be of relevance to leaky solid-state devices. Reference [4] indeed shows that conductive regions are likely to appear in the central regions of capacitive structures, where insulation between the plates is susceptible to structural defects. We note that under the extremes of an avalanche breakdown one would have a highly conductive path between the plates.

Given the azimuthally symmetric geometry of the problem, the task is to calculate the electromagnetic fields generated at an arbitrary radius inside the capacitor. To do that along an initially pedagogical way, one first uses the simplifying hypothesis of a fully uniform electric field between the plates. The current is calculated as one takes the wired capacitor as a simple RC circuit, and the magnetic field is obtained with help of Ampère's law, with the symmetric source provided by the electric and displacement currents.

The present work is concerned with the validity of this approach. We examine in more detail how far can we take the uniformity of the electric field as a good approximation. The conclusion is that the approach is accurate while the conductivity $g$ is small enough that the electric field can diffuse freely into the conducting wire. However, as the conductivity increases and electric field lines are expelled from the conductor, the system becomes increasingly inhomogeneous. This is demonstrated by panel (a) of Fig. 2 which shows the clear birth of an inhomogeneous and oscillatory mode as one goes past the transition value for $g$. A brief analysis of inherently nonuniform modes is also performed. In a way, as the conductivity grows what is seen is a transition between a dominant $\mathrm{RC}$ mode to a dominant LC mode of a RLC series circuit.

The present work does not look at certain features, as for instance nonuniformities along the capacitor axis, and finite conductivity of the capacitor plates. Despite these limitations, our model provides a way to determine the range of values for $g$ where the discharge is uniform across the axis, and what can be expected when one goes beyond the transition point.

\section{Acknowledgments}

This work is supported by CNPq and FAPERGS, Brazil, and by the Air Force Office of Scientific Research (AFOSR), USA, under the grant FA9550-121-0438. The author thanks fruitful discussions with Renato Pakter and the suggestions of an anonymous reviewer.

\section{References}

[1] Laboratory Booklet, General Physics II (PHYS182), Electricity and Magnetism for Engineering, IFUFRGS (on demand) (2013).

[2] F.L.H. Wolfs, http://teacher.nsrl.rochester edu/phy122/Lecture_Notes/Chapter35/ chapter35.html, (2013).

[3] Physics.Stackexchange.Com, http://physics stackexchange.com/questions/156259/theelectric-field-in-wires-in-a-circuit, (2016).

[4] E. Miranda, D. Jiménez, J. Suñé, E. O’Connor, S. Monaghan, I. Povey, K. Cherkaoui and P.K. Hurley, J. Vac. Sci. Technol. B 31, 01A107 (2013).

[5] J.R. Reitz, F.J. Milford and R.W. Christy, Foundations of Electromagnetic Theory (Addison-Wesley Publishing Company, Boston, 1980).

[6] R.P. Feynman, R.B. Leighton and M. Sands The Feynman's Lectures on Physics, Vol. II (AddisonWesley Publishing Company, Inc., London, 1964).

[7] D.S. Weile, https://www.eecis.udel edu/ weile/ELEG648Spring06/Resources/ Cylindrical.pdf (2006).

[8] G.B. Arfken and H.J. Weber, Mathematical Methods for Physicists (Elsevier Academic Press, San Diego, 2005).

[9] See https://en.wikipedia.org/wiki/ Dielectric_los Dielectric Loss, (2006).

[10] W.C. Elmore and M.A. Heald, Physics of Waves (Dover Publications, Inc., New York, 1985).

[11] J.D. Jackson, Classical Electrodynamics (John Wiley \& Sons, New York, 1975).

[12] Y. Levin, F.L. da Silveira and F.B. Rizzato, Am. J. Phys. 74, 815 (2006).

[13] G. Donoso, C.L. Lader and P. Martin, Am. J. Phys 79, 193 (2011).

[14] B. Irvine, M. Kemnetz, A. Gangopadhyaya and T. Ruubel, Am. J. Phys. 82, 273 (2014).

[15] Y. Levin and F.B. Rizzato, Phys. Rev. E 74, 066605 (2006). 